\title{
Erratum: Long-wavelength phonons in the crystalline and pasta phases of neutron-star crusts [Phys. Rev. C 97, 065805 (2018)]
}

\author{
David Durelø and Michael Urban
}

(Received 26 May 2020; published 3 August 2020)

DOI: 10.1103/PhysRevC.102.029901

Our article contains a couple of typographical errors and a mistake which affects the results for one of the phonons in the spaghetti phase. The sum of Coulomb and surface energies per volume Eq. (27) is missing a factor of $u^{2}$ and should read

$$
\varepsilon_{\mathrm{C}+\mathrm{S}}=\frac{\pi}{2} e^{2} n_{p, 2}^{2} R_{\mathrm{WS}}^{2} u^{2}(-1+u-\ln u)+\frac{2 \sqrt{u} \sigma}{R_{\mathrm{WS}}} .
$$

In the text following this equation, the variation of the size of the cell is missing a factor of $\frac{1}{2}$, so it should read $\delta R_{\mathrm{WS}}=\frac{1}{2} R_{\mathrm{WS}} \nabla_{\perp} \xi_{\perp}$.

The last typographical error concerns the last paragraph (p. 7) of Sec. V A where $K_{3}$ should be replaced by $K_{1}$.

Whereas these typographical errors do not affect the correctness of the calculations, this is not the case for the following mistake. There has been confusion with the coefficients $B$ defined in Eqs. (28)- (30), which are, in reality,

$$
\begin{aligned}
& B_{11}=\frac{1}{4}(-2+4 u) C_{0}, \\
& B_{13}=\frac{1}{4}(-4+6 u) C_{0}, \\
& B_{33}=\frac{1}{4}(-9+11 u-3 \ln u) C_{0} .
\end{aligned}
$$

This error affects only the lowest mode in the spaghetti phase. Figure 4 shows the velocities of the phonons of the spaghetti phase for the same parameters as in Fig. 4 in the original article. The qualitative behavior of the modes does not change only the velocity of the lowest mode (red lines) is a little higher.

The change in velocity of the lowest mode affects the specific heat associated with it. Figure 5 shows the new temperature dependence of the contributions of the different modes to the specific heat. Compared to Fig. 5 of the article, the contribution proportional to $T$ of the lowest transverse phonon (red line) is roughly reduced by a factor of 2 , and it is, now, approximately one half of the electron contribution.

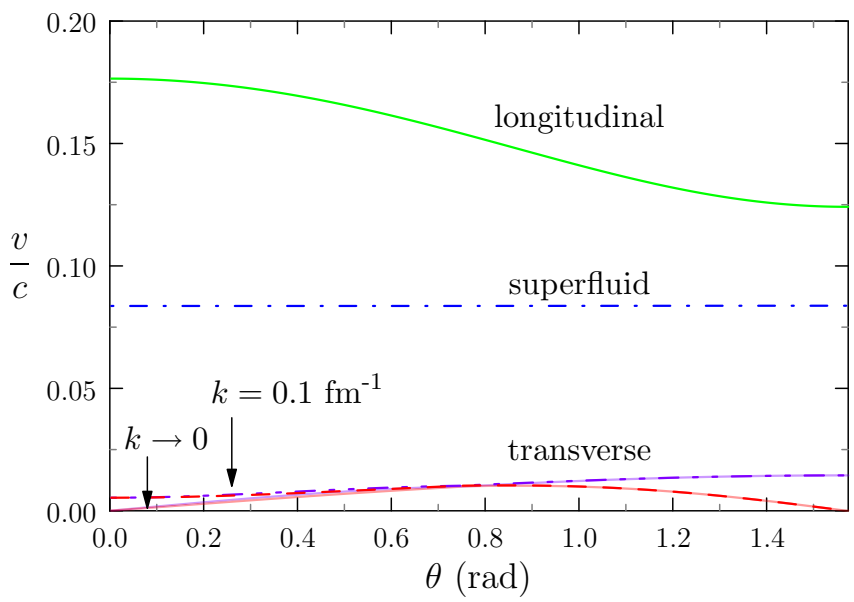

FIG. 4. Angle dependence of the velocities of the four phonons in the spaghetti phase. The parameters are given in Table I of the article. 


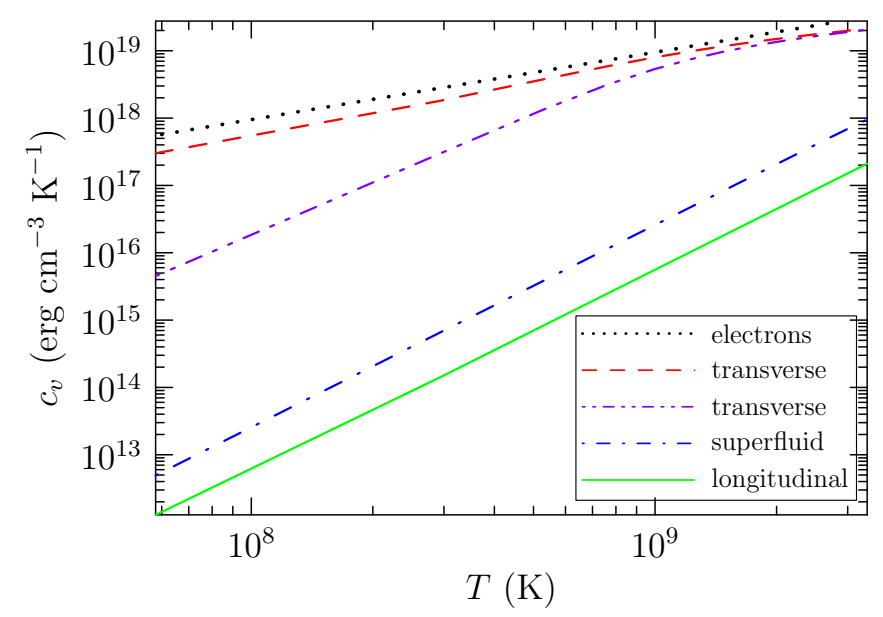

FIG. 5. Temperature dependence of the contributions to the specific heat of the spaghetti phase corresponding to the four phonons shown in Fig. 4. 